\title{
Statistics of active versus passive advections in magnetohydrodynamic turbulence
}

\author{
Thomas Gilbert* \\ Institut Non-Linéaire de Nice, CNRS, Université de Nice, 1361 Route des Lucioles, 06560 Valbonne, France \\ Dhrubaditya Mitra ${ }^{\dagger}$ \\ Centre for Condensed Matter Theory, Department of Physics, Indian Institute of Science, Bangalore 560012 , India
}

\begin{abstract}
Active turbulent advection is considered in the context of magnetohydrodynamics. In this case, an auxiliary passive field bears no apparent connection to the active field. The scaling properties of the two fields are different. In the framework of a shell model, we show that the two-point structure function of the passive field has a unique zero mode, characterizing the scaling of this field only. In other words, the existence of statistical invariants for the decaying passive field carries no information on the scaling properties of the active field.
\end{abstract}

In the context of turbulent advection, the understanding of fluid turbulence has greatly improved in the recent years [1]. The anomalous scaling has been shown to be universal and connected to the existence of statistical integrals of motion [2]. In Ref. [3], it was shown that the statistically conserved structures of decaying passive turbulence dominate the statistics of forced turbulence, thus offering a rather general framework for understanding the universality of anomalous scaling in forced turbulence.

Let $\phi$ be a decaying field transported by a stationary turbulent flow. The linearity of the advection implies the following relation for the correlation functions:

$$
\begin{aligned}
\left\langle\phi\left(\vec{r}_{1}, t\right) \cdots \phi\left(\vec{r}_{N}, t\right)\right\rangle= & \int d \underline{\vec{q}} P^{(N)}\left(\underline{\vec{r}}, t \mid \underline{\vec{q}}, t_{0}\right) \\
& \times\left\langle\phi\left(\vec{q}_{1}, t_{0}\right) \cdots \phi\left(\vec{q}_{N}, t_{0}\right)\right\rangle,
\end{aligned}
$$

where we used the compact notation $\vec{r} \equiv \vec{r}_{1}, \ldots, \vec{r}_{N}$ to denote a collection of $N$ position vectors. Equation (1) tells us there exists a linear operator $\mathcal{P}^{(N)}$ that propagates the $n$th order correlation function from time $t_{0}$ to time $t$. Without fresh input, that is, in the absence of forcing, the correlation functions of $\phi$ decay due to dissipative effects. Nevertheless, as conjectured in Ref. [3], there exist special functions $Z^{(N)}$ that are left eigenfunctions of eigenvalue 1 of the operator $\mathcal{P}^{(N)}$,

$$
Z^{(N)}(\underline{\vec{r}})=\int d \underline{\vec{q}} \mathcal{P}^{(N)}\left(\underline{\vec{q}}, t \mid \underline{\vec{r}}, t_{0}\right) Z^{(N)}(\underline{\vec{q}}),
$$

such that

$$
I^{(N)}(t)=\int d \underline{\underline{r}} Z^{(N)}(\underline{\vec{r}})\left\langle\phi\left(\vec{q}_{1}, t\right) \cdots \phi\left(\vec{q}_{N}, t\right)\right\rangle
$$

is preserved in time. $I^{(N)}$ and $Z^{(N)}$ are respectively called a statistical integral of motion and a statistically preserved structure of order $N$, also referred to as zero modes [15].

\footnotetext{
*Electronic address: thomas.gilbert@inln.cnrs.fr

${ }^{\dagger}$ Electronic address: dhruba@physics.iisc.ernet.in
}

Now, consider the same passive advection problem with an external forcing, such that the system reaches a stationary state. Define the correlation function of $\phi$ in that stationary state to be

$$
F^{(N)}(\underline{\vec{r}})=\left\langle\phi\left(\vec{r}_{1}, t\right) \cdots \phi\left(\vec{r}_{N}, t\right)\right\rangle_{f}
$$

where the symbol $\langle\cdot\rangle_{f}$ denotes averaging over the statistical stationary state. It was conjectured in Ref. [3], that the anomalous part of $F^{(N)}(\vec{r})$ is dominated by the leading zero modes of the decaying problem, i.e., $Z^{(N)} \sim F^{(N)}$. The conjecture was verified in the context of a shell model for passive scalar advection.

In subsequent studies, it was discovered that the existence of statistical invariants of the motion for passive turbulence may help us understand the statistics of active turbulence, a case where the advected quantity affects the dynamics of the advecting field. In Refs. [4-6], the case of thermal convection in the Boussinesq approximation was studied. There it was shown that the scaling of the active field is also dominated by the statistically preserved structures of auxiliary passive fields. It is yet unclear how general this connection between the statistics of active and auxiliary passive fields is. The case of two-dimensional magnetohydrodynamics is revealing. Indeed, in this case active and passive fields have very different scaling behaviors. As shown in Ref. [7], the transported fields cascade in different directions. It is therefore unexpected that the statistics of the auxiliary passive field holds information on the statistics of the active field. The two fields have different scaling properties. In Ref. [6], despite this difference, the claim was made that the analogy does hold in the sense that there exist subleading zero modes of the propagator of the correlation functions of the auxiliary passive field with the scaling of the correlation functions of the active field.

The purpose of this paper is to show that this is actually not the case. To this end, we will limit our investigation to the case of the second order structure functions of a shell model of two-dimensional magnetohydrodynamic turbulence. The case of the second order structure function is the simplest one. Because of the absence of geometry, its scaling 
is nonanomalous $[2,8,9]$, which in the language of zero modes implies nondegeneracy, that is, there is a unique conserved structure associated to the two-point statistical invariant. The same holds in the language of shell models, where the only two point function of a passive scalar field $\theta_{n}$ associated to the scale $k_{n}$ is $\left\langle\left|\theta_{n}\right|^{2}\right\rangle$, in contrast to higher order structure functions, e. g., the fourth order for which we have contributions from $\left\langle\left|\theta_{n}\right|^{4}\right\rangle,\left\langle\left|\theta_{n-1}\right|^{2}\left|\theta_{n+1}\right|^{2}\right\rangle$, etc. Using methods similar to those used in Ref. [10], we will construct the operator propagating the second order structure functions, and will demonstrate that the auxiliary passive field has the same statistical integral of motion as other shell models of passive advection. It will be inferred that there is no subleading zeromode with the scaling of the (active) magnetic field.

In analogy to other models [11-13], the following two sets of equations generalize the usual Sabra shell model [14] for the turbulent velocity field to magnetohydrodynamic turbulence (we omit dissipative terms):

$$
\begin{aligned}
\frac{d u_{n}}{d t}= & i\left[k_{n+1}\left(u_{n+1}^{*} u_{n+2}-b_{n+1}^{*} b_{n+2}\right)\right. \\
& -(\epsilon+1) k_{n}\left(u_{n-1}^{*} u_{n+1}-b_{n-1}^{*} b_{n+1}\right) \\
& \left.-\epsilon k_{n-1}\left(u_{n-2} u_{n-1}-b_{n-2} b_{n-1}\right)\right]+f_{n}, \\
\frac{d b_{n}}{d t}=i[ & -(\epsilon+\delta) k_{n+1}\left(u_{n+1}^{*} b_{n+2}-b_{n+1}^{*} u_{n+2}\right) \\
+ & \delta k_{n}\left(u_{n-1}^{*} b_{n+1}-b_{n-1}^{*} u_{n+1}\right) \\
+ & \left.(\delta-1) k_{n-1}\left(u_{n-2} b_{n-1}-b_{n-2} u_{n-1}\right)\right]+f_{n}^{\prime} .
\end{aligned}
$$

Here $u_{n}$ (the velocity field) and $b_{n}$ (the magnetic field) are complex variables defined on a discrete set of shells indexed by the integer $n$ whose associated wavenumber $k_{n}=k_{0} \lambda^{n}$, $\lambda>1$ (hereafter taken to be $\lambda=2$ ). $f_{n}$ and $f_{n}^{\prime}$ are two forcing terms which are taken to be stochastic white noises with identical statistics and concentrated on a limited number of neighboring shells ( $n=5,6,7$ in our numerical experiments). The model's parameters $\epsilon$ and $\delta$ are conveniently parametrized in the following way. The three-dimensional (3-D) model for which $\epsilon<0$ reads $(\alpha>0)$

$$
\begin{aligned}
& \epsilon=-\lambda^{-\alpha}, \\
& \delta=\left(1+\lambda^{\alpha}\right)^{-1} \quad \text { (3D model). }
\end{aligned}
$$

The two-dimensional problem on the other hand has $\epsilon>0$ and reads

$$
\begin{aligned}
& \epsilon=\lambda^{-\alpha} \\
& \delta=-\left(\lambda^{\alpha}-1\right)^{-1} \quad \text { (2D model). }
\end{aligned}
$$

Correspondingly, we have the following quadratic dynamical invariants (i.e., time-invariant in the limit of zero viscosity and zero external forcing),

$$
\begin{gathered}
E=\sum_{n}\left(\left|u_{n}\right|^{2}+\left|b_{n}\right|^{2}\right) \quad \text { (total energy), } \\
K=\sum_{n} \operatorname{Re}\left(u_{n}^{*} b_{n}\right) \quad \text { (cross helicity), }
\end{gathered}
$$

$$
H=\sum_{n} \operatorname{sgn}(\delta)^{n} k_{n}^{-\alpha}\left|b_{n}\right|^{2} \quad \text { (magnetic helicity) }
$$

Thus, in both two- and three-dimensional models, Eqs. (5) and (6) have one single free parameter, $\alpha>0$. The two- and three-dimensional models actually have very different dynamical behaviors, see Ref. [11]. Only the two-dimensional model can sustain a stationary state and we will limit ourselves to this case. As dimensional analysis shows [6], the conservation of the first two invariants implies that $u_{n}$ and $b_{n}$ must both have Kolmogorov scalings, $\left\langle\left|u_{n}\right|^{2}\right\rangle,\left\langle\left|b_{n}\right|^{2}\right\rangle \sim k_{n}^{-2 / 3}$, for which the corresponding fluxes are constant. This is indeed what has been measured for similar models [11], where both fields appear to display the same anomalies. Further, as shown in Ref. [6], the conservation of the third invariant allows for another scaling, $\left\langle\left|b_{n}\right|^{2}\right\rangle \sim k_{n}^{\alpha-2 / 3}$, for which the magnetic helicity flux is constant. But since this scaling is incompatible with the conservation of the two other invariants, it is not relevant to the statistics of the magnetic field.

However, if one considers a passive auxiliary field obeying an equation identical to Eq. (6) for the two-dimensional case,

$$
\begin{aligned}
\frac{d a_{n}}{d t}= & i\left[\lambda^{-\alpha}\left(\lambda^{\alpha}-1\right)^{-1} k_{n+1}\left(u_{n+1}^{*} a_{n+2}-a_{n+1}^{*} u_{n+2}\right)\right. \\
& -\left(\lambda^{\alpha}-1\right)^{-1} k_{n}\left(u_{n-1}^{*} a_{n+1}-a_{n-1}^{*} u_{n+1}\right) \\
& \left.-\lambda^{\alpha}\left(\lambda^{\alpha}-1\right)^{-1} k_{n-1}\left(u_{n-2} a_{n-1}-a_{n-2} u_{n-1}\right)\right],
\end{aligned}
$$

the only relevant invariant is the equivalent of the magnetic helicity Eq. (11), to which a constant flux is associated. Thus the dimensional scaling $\left\langle\left|a_{n}\right|^{2}\right\rangle \sim k_{n}^{\alpha-2 / 3}$ is expected to be observed. And that is indeed what was found in Ref. [6].

Notice though that the linearity of Eq. (12) allows for the substitution $\psi_{n}=\lambda^{-\alpha n / 2} a_{n}$. With this new variable, Eq. (12) takes the form

$$
\begin{aligned}
\frac{d \psi_{n}}{d t}= & i\left[A\left(k_{+1} u_{n+1}^{*} \psi_{n+2}+k_{n-1} \psi_{n-2} u_{n-1}\right)+B\left(k_{n+1} \psi_{n+1}^{*} u_{n+2}\right.\right. \\
& \left.\left.-k_{n} \psi_{n-1}^{*} u_{n+1}\right)+C\left(k_{n} u_{n-1}^{*} \psi_{n+1}+k_{n-1} u_{n-2} \psi_{n-1}\right)\right],
\end{aligned}
$$

which describes the advection of a scalar for which the quadratic invariant is $\Sigma_{n}\left|\psi_{n}\right|^{2}$, which is similar to the shell model with only nearest neighbor interaction considered in Refs. $[3,10]$. The coefficients in Eq. (12) correspond to the choice $A=\left(\lambda^{\alpha}-1\right)^{-1}, B=-\lambda^{-\alpha / 2}\left(\lambda^{\alpha}-1\right)^{-1}$, and $C=-\lambda^{\alpha / 2}\left(\lambda^{\alpha}-1\right)^{-1}$. This shows that as far as shell models are concerned, the difference between a passively advected vector and passively advected scalar is just a numerical factor. Henceforth we do not make a distinction between the two and use the name "passive field" for both.

Consider now the equivalent of Eq. (1) for the propagation of the second order structure functions $\left\langle\left|\theta_{n}\right|^{2}\right\rangle$ in the decaying problem-including dissipative terms on the righthand side of Eq. (13). Following notations similar to those used in Ref. [10], we can write the equation of motion for $\psi_{n}$ under the form 


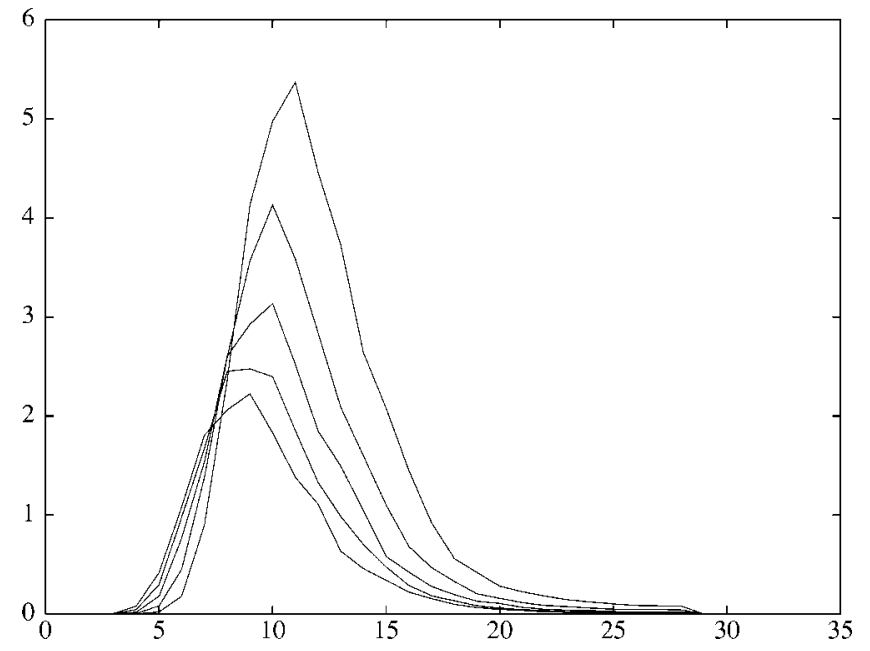

FIG. 1. The elements of $\mathcal{P}_{n, 20}^{(2)}$ for the scalar field Eq. (13) advected by the magnetohydrodynamic field Eqs. (5) and (6), where the parameter was chosen to be $\alpha=2$. The times displayed are .4, $.55, .7, .85$, and 1 , respectively (measured in the natural time units of the model). The horizontal axis corresponds to the shell numbers. The units on the vertical scale are arbitrary. The simulation was done using a total of 35 shells, with the first shell wave number $k_{0}=1 / 16$ and a shell spacing of $\lambda=2$. All the fields were dissipated on the small scales with a term $\nu k_{n}^{2}$, with $\nu=10^{-12}$. The advecting fields were forced on shells 5-7 with white delta correlated noise of amplitudes $1 / \sqrt{2}, 1 / 2 \sqrt{2}$, and $1 / 4$, respectively. Moreover, the phase of the forcing on shell 7 was taken to be equal to the sum of the phases of the forcings on shells 5 and 6 .

$$
\frac{d \psi_{n}}{d t}=\mathcal{L}_{n, m} \psi_{m}
$$

with the solution

$$
\begin{aligned}
\psi_{n}(t) & =\mathrm{T}^{+}\left\{\exp \left[\int_{t_{0}}^{t} d s \mathcal{L}(s)\right]\right\}_{n, m} \psi_{m}\left(t_{0}\right) \\
& \equiv \mathcal{R}_{n, m}\left(t \mid t_{0}\right) \psi_{m}\left(t_{0}\right)
\end{aligned}
$$

( $\mathrm{T}^{+}$denotes the time ordering operator). Letting

$$
\mathcal{P}_{n, m}^{(2)}\left(t \mid t_{0}\right) \equiv\left\langle\mathcal{R}_{n, m}\left(t \mid t_{0}\right) \mathcal{R}_{n, m}^{*}\left(t \mid t_{0}\right)\right\rangle,
$$

the propagation of second order structure functions obeys the following equation:

$$
\left\langle\left|\psi_{n}(t)\right|^{2}\right\rangle=\sum_{m} \mathcal{P}_{n, m}^{(2)}\left(t \mid t_{0}\right)\left\langle\left|\psi_{m}\left(t_{0}\right)\right|^{2}\right\rangle
$$

The form of the operator $\mathcal{P}^{(2)}$ was discussed in Ref. [10]. It is a matrix whose elements can be obtained by propagating an initial condition concentrated at a given shell. Similar considerations hold for the models considered here. In Fig. 1 we show these elements for successive times, starting from an initial condition at shell 20. The model we used is Eq. (13) for the parameters corresponding to Eq. (12) and advected by the magnetohydrodynamic fields Eqs. (5) and (6). The parameters of the simulation are given in the figure caption.

In analogy to Ref. [3], the statistical invariant $I^{(2)}$ for the passive scalar field is

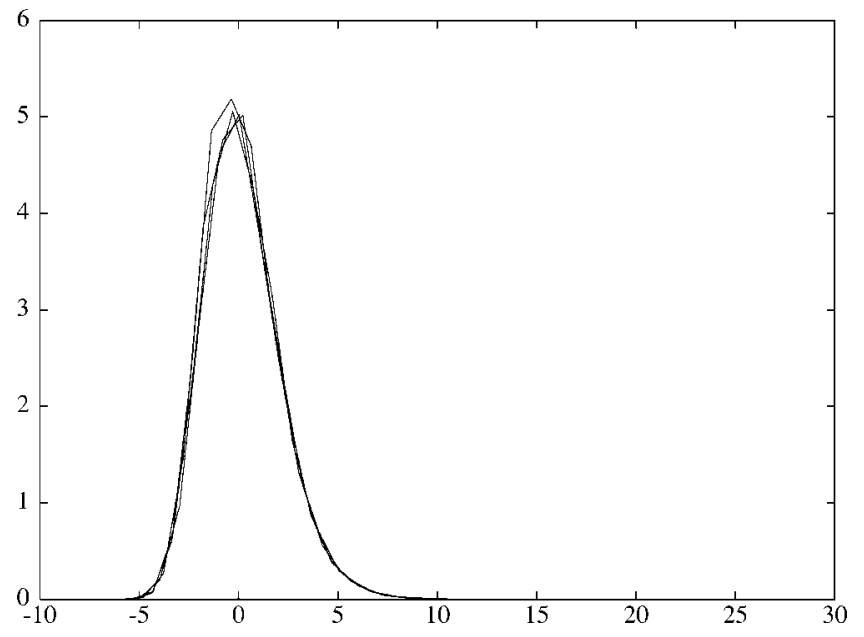

FIG. 2. The curves of Fig. 1 collapsed according to Eq. (18).

$$
I^{(2)}(t)=\sum_{n} Z_{n}^{(2)}\left\langle\left|\psi_{n}(t)\right|^{2}\right\rangle
$$

where $Z_{n}^{(2)}$ is a left eigenfunction of the operator $\mathcal{P}^{(2)}$, with the scaling of the second order structure function of the forced problem. The invariance of $I^{(2)}$ is most easily demonstrated by re-scaling the decaying second order objects according to Eq. (18). The curves indeed collapse if the ordinate is shifted with the appropriate time dependence. This is shown in Fig. 2 and is analogous to Fig. 4 in Ref. [10]. Thus in the language of the passive magnetohydrodynamic model Eq. (12), we have that

$$
\sum_{n}\left\langle\left|a_{n}\right|^{2}\right\rangle_{f} / k_{n}^{\alpha}\left\langle\left|a_{n}\right|^{2}(t)\right\rangle / k_{n}^{\alpha}
$$

is a statistical integral of motion. To claim that the scaling of the magnetic field structure function $\left\langle\left|b_{n}\right|^{2}\right\rangle$ is a subleading zero mode of $\mathcal{P}^{(2)}$ is equivalent to claiming that

$$
\sum_{n}\left\langle\left|b_{n}\right|^{2}\right\rangle / k_{n}^{\alpha}\left\langle\left|a_{n}\right|^{2}(t)\right\rangle / k_{n}^{\alpha}
$$

is a statistical integral of motion. Clearly, in view of Fig. 2 this cannot be the case and the collapse will not occur should the scaling exponent of $Z^{(2)}$ be replaced by another one [16]. In this line of thought, it is perhaps worthwhile pointing out that the collapse as seen in Fig. 2 would not be possible should there be zero modes with distinct scaling exponents. Indeed, as seen from Eq. (18), the collapse occurs provided $\left\langle\left|\psi_{n}(t)\right|^{2}\right\rangle$ "falls" precisely on the right eigenmode with a scaling identical to $Z_{n}^{(2)}$.

To conclude, we emphasize that the linearity of the passive advection models assigns them to a narrow class of equivalence. The passive magnetohydrodynamic model is in fact equivalent to a scalar advection model for which the 
statistical invariants have already been investigated in some detail [10]. In magnetohydrodynamics, active and passive fields have different scaling properties. The arguments that were used in the framework of thermal convection to account for the anomalous scaling of the active field in terms of a passive auxiliary one do not carry over to magnetohydrodynamics. The claim that one can nevertheless account for the scaling of the (active) magnetic field by considering subleading zeromodes of the operators propagating the decaying correlation functions was proven wrong. In view of the form of the second order propagator, it is clear that there is no zero mode but the one whose scaling is that of the passive field.
The authors gratefully acknowledge discussions with A. Celani, U. Frisch, and R. Pandit. T.G. also wishes to thank Y. Cohen, I. Procaccia, and A. Pumir. D.M. thanks D. Vincenzi. This work was partially done while D.M. was visiting the Observatoire de la Côte d'Azur in Nice. The support of CEFIPRA under Project No 2404-2 is acknowledged. T.G. acknowledges financial support from the European Union under Contract Nos. HPRN-CT-2000-00162 and HPRN-CT2002-00300. D.M. also wishes to thank CSIR India for financial support.
[1] G. Falkovich, K. Gawedzki, and M. Vergassola, Rev. Mod. Phys. 73, 913 (2001).

[2] A. Celani and M. Vergassola, Phys. Rev. Lett. 86, 424 (2001).

[3] I. Arad, L. Biferale, A. Celani, I. Procaccia, and M. Vergassola, Phys. Rev. Lett. 87, 164502 (2001).

[4] A. Celani, T. Matsumoto, A. Mazzino, and M. Vergassola, Phys. Rev. Lett. 88, 054503 (2002).

[5] E. S. C. Ching, Y. Cohen, T. Gilbert, and I. Procaccia, Europhys. Lett. 60, 369 (2002).

[6] E. S. C. Ching, Y. Cohen, T. Gilbert, and I. Procaccia, Phys. Rev. E 67, 016304 (2002).

[7] A. Celani, M. Cencini, A. Mazzino, and M. Vergassola Phys. Rev. Lett. 89, 234502 (2002).

[8] O. Gat and R. Zeitak, Phys. Rev. E 57, 5511 (1998).

[9] D. Bernard, K. Gawedzki, and A. Kupiainen, J. Stat. Phys. 90, 519 (1998).

[10] Y. Cohen, T. Gilbert, and I. Procaccia, Phys. Rev. E 65, 026314 (2002).

[11] P. Giuliani, in Nonlinear MHD Waves and Turbulence, edited by T. Passot and P. L. Sulem, Lecture Notes in Physics Vol. 536 (Springer, New York, 1999).
[12] A. Basu, A. Sain, S. K. Dhar, and R. Pandit, Phys. Rev. Lett. 81, 2687 (1998).

[13] P. Frick and D. Sokoloff, Phys. Rev. E 57, 4155 (1998).

[14] V. S. L'vov, E. Podivilov, A. Pomyalov, I. Procaccia, and D. Vandembroucq, Phys. Rev. E 58, 1811 (1998).

[15] Note that Eq. (2) is not stricto sensu an eigenvalue problem; $\mathcal{P}^{(N)}$ is not defined on a compact space. Only in the case of scaling advecting field is it possible to write down a proper operator and identify the zero modes as left eigenmodes with unit eigenvalues [9].

[16] Our claim is that there is only one unique scaling exponent $\beta_{2}$ (which is the scaling exponent of $Z^{(2)}$ ) which will make the quantity $\Sigma_{n} k_{n}^{\beta_{2}} / k_{n}^{\alpha}\left\langle\left|a_{n}\right|^{2}(t)\right\rangle / k_{n}^{\alpha}$ a statistical integral of motion. This can be proved if the velocity driving the passive field is Kraichnan (i.e., Gaussian delta correlated in time), but for a generic velocity field we know no way to rigorously prove this. One can, however, argue that the statistical integral of motion should depend on the geometry of the correlation function and not on the details of the statistics of the advecting field. 